\title{
Editorial
}

\section{Intensive Care Research: Science to Practice, and from Bedside to Bench}

Intensive Care Medicine was first introduced in the late 1950s to monitor life-threatened patients in a minute-by-minute manner and provide special care by a group of medical specialists, nurses, therapists, and clinical pharmacists in dedicated medical care units. Since then, substantial progress has been made rapidly in the management of critically ill patients in monitoring, technologies, intervention and eventually the improvement of outcomes and quality of life. None of the outstanding advancement can be achieved without continuous high-quality research in intensive care medicine. It is evidently essential to have a multi-disciplinary team of basic scientists, clinician-scientists, bioengineers and trainees to get engaged in cooperation and collaboration in order to achieve the ultimate goal of our research-improve people's health. Basic research is fundamental for our understanding of the mechanistic insights at cellular and molecular levels, physiopathology and pharmacology for development of effective therapies

\section{Haibo Zhang*}

Interdepartmental Division of Critical Care Medicine; Department of Anesthesiology and Pain Medicine; and Department of Physiology, University of Toronto, ON, Canada

Keenan Research Center for Biomedical Science, Li Ka Shing Knowledge Institute, St. Michael's Hospital, Unity Health Toronto, Room 619, 30 Bond Street, Toronto, ON, M5B 1W8, Canada in critically ill patients. For example, the finding of ventilatorinduced lung injury made in animal models with high tidal volumes was successfully translated into protective ventilation guidelines at bedside practice and saved hundreds of thousands of critically ill patients worldwide. Clinical research on therapeutic and preventive interventions is largely based on the appropriate methodology to minimize random and systematic errors, the interplay of medical prognosis and patient autonomy, and the clinical risks and patient safety in a clinical research setting. Comprehensive high-quality research results will provide the right format for the decision maker and eventually be implemented toward patient care. In response to pandemic like the COVID-19, the need for high-quality basic and clinical research is greater and sounder now than ever. Input from experts in the fields dedicated to translating scientific research from bench to bedside and back is the mission of the Intensive Care Research. 